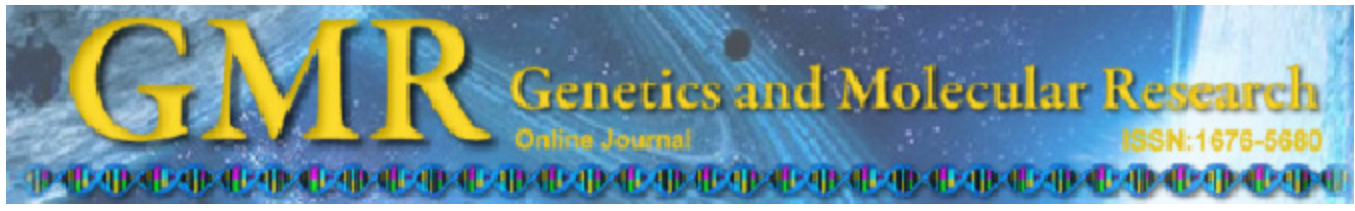

\title{
Analysis of interaction between Bmhrp28 and BmPSI in sex-specific splicing of Bombyx mori Bmdsx gene
}

\author{
X.F. Zha*, M. Zhao*, C.Y. Zhou, H.Z. Guo, P. Zhao, Z.H. Xiang and \\ Q.Y. Xia
}

State Key Laboratory of Silkworm Genome Biology, College of Biotechnology, Southwest University, Chongqing, China

*These authors contributed equally to this study.

Corresponding author: Q.Y. Xia

E-mail: xiaqy@swu.edu.cn

Genet. Mol. Res. 13 (3): 5452-5462 (2014)

Received June 24, 2013

Accepted November 29, 2013

Published July 24, 2014

DOI http://dx.doi.org/10.4238/2014.July.24.25

\begin{abstract}
Bombyx mori BmHRP28 and BmPSI, which belong to the family of RNA-binding proteins, have been identified binding to the female-specific exon 4 of the sex-determining gene $B m d s x$ pre-mRNA. However, the relationships between BmHRP28 and BmPSI still remain unclear. In this study, we carried out yeast two-hybrid (Y2H) and coimmunoprecipitation (Co-IP) analyses to address them. Y2H analysis showed that there was little or no direct binding between the BmHRP28 and BmPSI proteins. Also, the Co-IP experiments revealed that BmHRP28 and BmPSI coexisted in a multiprotein complex. Our results suggested that BmHRP28 and BmPSI form a muliprotein complex to regulate the splicing of Bmdsx pre-mRNA, but are not directly bound to each other. In an effort to find other regulatory factors in the multiprotein complex, we constructed a silkworm Y2H cDNA library of male early embryo. By Y2H screening, we identified an RNA-binding protein BmSPX, a putative component of the spliceosome, binding to BmPSI. These results indicated that BmHRP28 and BmPSI make up a
\end{abstract}


spliceosome complex to regulate $B m d s x$ splicing and that BmSPX is another potential protein involved in this process. Our study provides some clues to better understand the mechanism of sex determination in the silkworm.

Key words: Bombyx mori; Sex determination; Bmhrp28; BmPSI

\section{INTRODUCTION}

Sex dimorphism is one of the fascinating scientific issues in the biosphere. Although there are only two sexes in the world, the genetic system for sex determination is highly diversified. The fruit fly, Drosophila melanogaster, is a model organism. Somatic sex determination and differentiation in Drosophila is controlled by a cascade of genes (Baker, 1989; Cline, 1989; Steinmann-Zwicky et al., 1990). The primary genetic signal for sex determination is the X:autosome ratio (Cline, 1993; Cline and Meyer, 1996). In the sex determination pathway, the double-switch gene doublesex $(d s x)$ is the most important target. Through alternative splicing, the $d s x$ pre-mRNA produces male-specific (DSXM) and female-specific (DSXF) proteins (Baker and Wolfner, 1988; Burtis and Baker, 1989). In females, the femalespecific splicing of the $d s x$ pre-mRNA depends on the activity of tra and transformer 2 (tra2) (Inoue et al., 1992; Tian and Maniatis, 1993; Schutt and Nothiger, 2000). The transformer (tra) gene regulates female-specific splicing of $d s x$ and has no known function in males. The two products of $d s x$, DSXM and DSXF, activate the male and female differentiation genes, respectively (Baker et al., 1989; Slee and Bownes, 1990).

The silkworm, Bombyx mori, is a female-heterogametic insect ( $\mathrm{ZZ}$ in males, $\mathrm{ZW}$ in females). The feminizing gene ( $F e m$ ) on the $\mathrm{W}$ chromosome of $B$. mori is the dominant factor in determining the female sex (Hashimoto, 1933). Although the mechanism is distinct between B. mori and D. melanogaster, the homolog of doublesex $(B m d s x)$ is a double-switch gene in the final step of the sex determination cascade in B. mori as the function of $d s x$ in D. melanogaster. Bmdsx pre-mRNA is also alternatively spliced in males and females to produce malespecific (BmDSXM) and female-specific (BmDSXF) proteins (Ohbayashi et al., 2001; Suzuki et al., 2003, 2005). Thus, the upstream regulatory genes between $B m d s x$ and $d s x$ are different. The homologue of Drosophila PSI, BmPSI, was previously identified as the nuclear factor that specifically binds to Bmdsx and regulates the male-specific splicing of Bmdsx (Suzuki et al., 2008). Subsequently, a $30-\mathrm{kDa}$ protein was identified as Bmhrp28, a protein similar to the Drosophila splicing factor $h r p 48$, and it was shown that Bmhrp 28 recognizes specific sequence involved in the alternative splicing of Bmdsx pre-mRNA (Wang et al., 2009). In Drosophila, $P S I$ is an alternative splicing factor that regulates tissue-specific splicing, probably by cooperating with another splicing factor hrp48 (Siebel et al., 1994; Adams et al., 1997).

In Bombyx mori, BmPSI and Bmhrp 28 are alternative splicing factors that specifically bind to Bmdsx (Suzuki et al., 2008; Wang et al., 2009). In addition, since the splicing of premRNA needs many splicing factors (Lopez, 1998), there may be other factors regulating the $B m d s x$ splicing process. The relationships between BmPSI and Bmhrp 28 are not well known. In this study, we tested if there is a direct physical interaction between them. At the same time, we found another novel factor, Bmspx, bound to BmPSI through screening the yeast twohybrid (Y2H) cDNA library of the silkworm. 


\section{MATERIAL AND METHODS}

\section{Prokaryotic expression and polyclonal antibody preparation of BmPSI and Bmhrp28}

The coding regions of the BmPSI and Bmhrp 28 genes were amplified by PCR with pairs of primers containing Bam HI and XhoI (forward: 5'-CGCggatccATGAGTGATTATTC TTCTAT-3', reverse: 5'-CCGctcgagTCACTGCTGGTGGTCGGAGC-3') and NdeI and NotI sites (forward: 5'-GGAATTC catatgCGTATGAATCCAGACATGGA-3', reverse: 5'-ATAAGA ATgcggcegcCTAGCGCCTGTAGGGATGGT-3'), respectively. The amplified products were cloned into pET-MBP and pET28a vectors. The resultant plasmids were transformed into Escherichia coli Rosetta (DE3) cells and BL21 (DE3) cells. The approach of expressed BmPSI protein was as follows: a single colony was picked and transferred to fresh liquid media (containing $30 \mu \mathrm{g} / \mathrm{mL}$ kanamycin and $34 \mu \mathrm{g} / \mathrm{mL}$ chloromycetin) and grown at $37^{\circ} \mathrm{C}$ to an OD600 of 0.6-1.0 before induction with $0.2 \mathrm{mM}$ IPTG. After $4 \mathrm{~h}$ induction, the cells were harvested by centrifugation and expressed proteins were purified by affinity chromatography with an Econo-Pac chromatography column. The method for expression of BmHRP28 protein was similar to that for BmPSI. The expressed BmPSI protein was purified by the His binding protein purification kit (Merck, Germany).

The purified BmHRP28 protein was used for obtaining rabbit antibodies. An adult male rabbit was immunized with a mixture of $700 \mu \mathrm{g}$ purified protein and the same volume of complete Freund's adjuvant. Booster immunization was carried out 10 days later with the same dose of Freund's incomplete adjuvant. Two more boosters were then administered each week. Ten days later after the last booster, a blood sample from the ear vein was assayed for antibody response by ELISA. The BmPSI protein was used for obtaining antibodies by the same method as that of BmHRP28. The purification of polyclonal antibodies was carried out according to a standard protocol.

\section{Co-immunoprecipitation (Co-IP) and Western blot analysis}

Silkworm tissues were rinsed with PBS and homogenized in a glass homogenization tube, and lysed in cell lysis buffer. Lysates were clarified by centrifugation at $14,000 \mathrm{~g}$ at $4^{\circ} \mathrm{C}$ for $5 \mathrm{~min}$. The supernatant was precleared with $40 \mu \mathrm{L}$ of protein A-Sepharose beads (Beyotime, China) for $2 \mathrm{~h}$ at $4^{\circ} \mathrm{C}$. Appropriate amounts of rabbit anti-BmHRP28 were then added and the tubes incubated overnight at $4^{\circ} \mathrm{C}$. After washing with PBS buffer, bound proteins were eluted in sodium dodecyl sulfate (SDS) sample buffer and resolved by SDS-polyacrylamide gel electrophoresis (PAGE). The resolved proteins were transferred electrically to polyvinylidene fluoride membranes (Millipore, Germany). Detection of proteins was performed by incubation with rabbit anti-BmPSI polyclonal antibodies and anti-rabbit IgG (whole molecule)peroxidase antibody. The membranes were subsequently stained with chemiluminescent substrate with the aid of a Western chemiluminescent substrate kit.

\section{Y2H analysis}

In this research, the ORFs of BmPSI and Bmhrp28 were recombined into the twohybrid system vectors pDBLeu and pPC86 (Gibco BRL, USA), respectively. The vectors pPC86 and recombined pDBLeu-BmPSI, as well as pDBLeu and pPC86-Bmhrp28, were 
transferred into the yeast MaV203 cells for self-activation effect. In the absence of selfactivation, pDBLeu-BmPSI and pPC86-Bmhrp28 were co-transferred into the yeast MaV203 strain and incubated on SC-Lea-Trp, SC-Leu-Trp-Ura, and SC-Leu-Trp-His+3-AT plates at $30^{\circ} \mathrm{C}$, respectively. The positive colonies were replica-plated onto filter paper and immersed in liquid nitrogen. The frozen filter paper was saturated with Z-buffer containing X-Gal and incubated at $37^{\circ} \mathrm{C}$ for over $24 \mathrm{~h}$.

\section{Construction and screen of $\mathrm{Y} 2 \mathrm{H}$ library}

The silkworm strain sex-linked white egg was reared under standard conditions. Total RNA was extracted using TRIzol (Invitrogen, USA) from male early embryos (48, 60, 72, and $96 \mathrm{~h}$ after fertilization) of silkworm. mRNA was further isolated from a mixture of total RNAs of four samples. Following first strand cDNA synthesis, we used the CloneMiner cDNA Library Construction kit (Invitrogen, USA) to construct the entry library in pDONR222 (Invitrogen, USA). Serial dilutions were performed to calculate the number of primary clones. To examine the average clone lengths, we isolated plasmid DNA for 40 randomly selected clones from the library and detected the clone lengths using PCR. Inserts from the entry library were shuttled into the $\mathrm{Y} 2 \mathrm{H}$ prey vector, pDEST22 using LR Clonase II enzyme mix (Invitrogen) to create the pDEST22 (Y2H) library. To identify a novel factor in the multiprotein complex except Bmhrp28 and BmPSI, we carried out a screen in the Y2H library of silkworm early embryo by using the bait vector pDBLeu-BmPSI.

\section{RESULTS}

\section{Preparation of polyclonal antibodies to BmPSI and Bmhrp28}

The binding of BmPSI and Bmhrp28 specifically to the silkworm sex-determining gene Bmdsx was previously determined and analyzed (Suzuki et al., 2008; Wang et al., 2009). To confirm the relationship between BmPSI and Bmhrp28, recombinant BmPSI and BmHRP28 proteins were expressed in E. coli. The cDNA of BmPSI and Bmhrp 28 were ligated into pETMBP and pET28a plasmids to construct prokaryotic expression vectors called BmPSI/pET-MBP and Bmhrp28/pET28a, respectively. They were transformed into the expression strains Rosetta (DE3) and BL21 (DE3) and then induced by IPTG. Two recombinant proteins of 120 and $31 \mathrm{kDa}$ were obtained, respectively (Figure S1). The molecular weights were a little higher than that of the original proteins because of the added MBP-tag and 6xHis-tag. Using affinity chromatography and electroelution, respectively, we purified recombinant BmPSI and BmHRP28 (Figure 1), and then immunized male rabbits with the two proteins to obtain polyclonal antibodies.

\section{Interaction between BmPSI and Bmhrp28}

To identify the relationship between Bmhrp28 and BmPSI, we performed $\mathrm{Y} 2 \mathrm{H}$ and Co-IP assays. First, in the absence of self-activation using Y2H, the genes tested were cotransformed into yeast to determine the relationship between the target genes by detecting the three reporter genes (HIS3, URA3, LacZ). In this research, BmPSI and BmHRP28 as bait and prey protein were connected to the pDBLeu and pPC 86 vectors, respectively. In the absence 
of self-activation, pDBLeu-BmPSI and pPC86-Bmhrp 28 were co-transfected into yeast. As a result, there were no positive clones on the SC-Leu-Trp-His+3-AT and SC-Leu-Trp-Ura plates, and there was no positive result in the membrane detection of $L a c Z$. The expressions of the three reporter genes HIS3, URA3, LacZ were not detected (Figure 2). The results indicated that there was no or little direct binding between BmPSI and Bmhrp28.

(A)

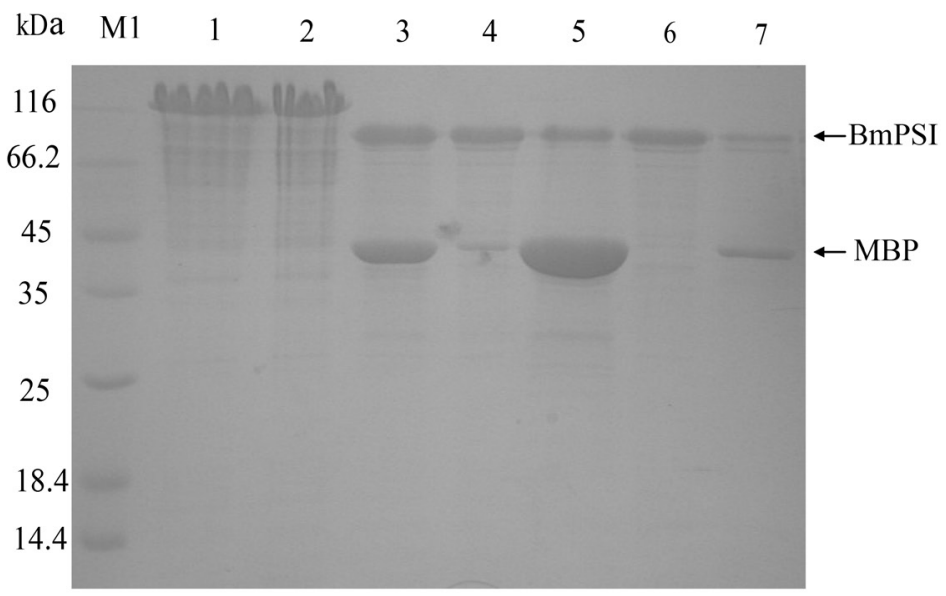

(B)

(C)
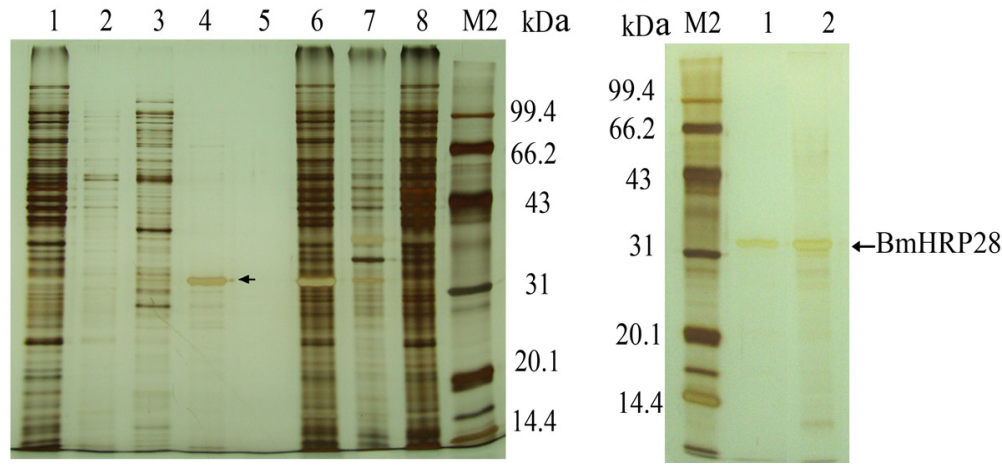

Figure 1. Purification of BmPSI and BmHRP28. Proteins were separated on a $12.5 \%$ SDS-PAGE and the gel was stained with Coomassie brilliant blue R250 and silver nitrate. A. The result of purified BmPSI protein. Lane $M 1=$ unstained protein molecular weight marker; lane $1=\mathrm{BmPSI} / \mathrm{pET}$-MBP was eluted with imidazole; lane 2 = changing buffer by desaliting; lane $3=$ digested by rTEV; lane $4=$ BmPSI protein after digestion (the first time); lane $5=$ eluted with imidazole after digestion (contain a small amount of BmPSI and a large number of MBP). Lane $6=$ BmPSI protein after digestion (the second time); lane $7=$ the second elution with imidazole after digestion (contain a small part of the BmPSI and MBP). B. Affinity chromatography of BmHRP28. Lane 1 = flow through; lane $2=$ washed by binding buffer; lane 3 = washed by wash buffer; lane $4=$ eluted by elute buffer; lane 5 = washed by strip buffer; lane $6=$ supernatant of the culture processed with ultrasonic, induced Bmhrp28/p28; lane $7=$ deposit of the culture processed with ultrasonic, induced Bmhrp28/p28; lane $8=$ deposit of the culture processed with ultrasonic, non-induced Bmhrp28/p28; lane M2 = protein molecular weight marker (low), the arrow indicates target protein. C. Electro-eluting result of BmHRP28. lane $M 2=$ protein molecular weight marker (low); lane $1=$ purified protein of BmHRP28 by electro-elution; lane 2 = affinity chromatography of BmHRP28, the arrow indicates target protein. 


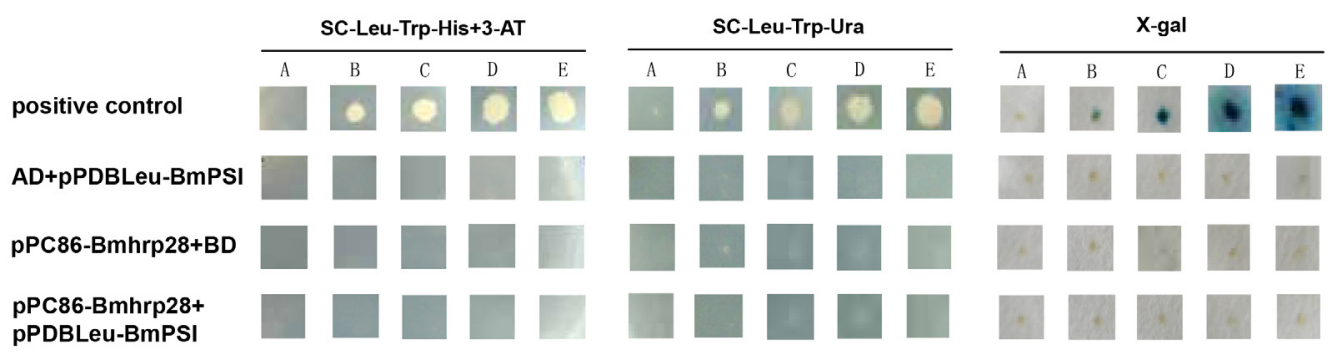

Figure 2. Analysis of interaction between Bmhrp28 and BmPSI by yeast two-hybrid. A. B. C. D. and E. Five control strains. The positive control shows that the intensity of control interaction gradually increased. AD+pPDBLeu-BmPSI and pPC86-Bmhrp28+BD were used as controls. pPC86-Bmhrp28+pPDBLeu-BmPSI represents both recombined plasmids were co-transfected into yeast MaV203. Yeast cells containing plasmids are grown up and selected on SC-LeuTrp-His+3-AT medium and SC-Leu-Trp-Ura medium. X-Gal represents the selection of yeast colonies by X-Gal assay.

To further analyze the potential interaction between BmPSI and Bmhrp28, we used Co-IP experiments. Anti-BmHRP28 antibody precipitated its antigen in the testis extract of B. mori and at the same time protein that interacted with BmHRP28 was also precipitated. As shown in Figure S2 , by SDS-PAGE and Western blotting, the BmHRP28 protein was deposited and identified by anti-BmHRP28 antibody. Anti-BmPSI antibody was then used to detect whether BmPSI was in the precipitation product. The assays showed that BmPSI was also detected by anti-BmPSI antibody in the precipitate which was formed by anti-BmHRP28 antibody (Figure 3). These results indicated that BmHRP28 and BmPSI formed the multiprotein complex. Considering the results of $\mathrm{Y} 2 \mathrm{H}$, we presumed that BmHRP28 and BmPSI formed the complex but did not bind directly.

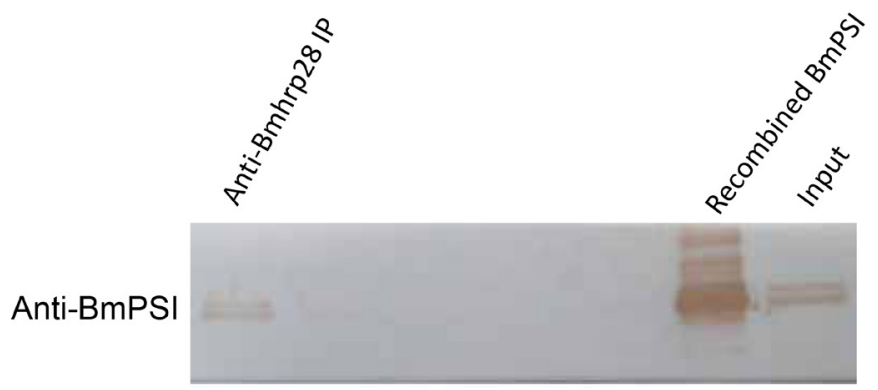

Figure 3. Co-immunoprecipitation (Co-IP) of BmPSI by BmHRP28. The IP product of BmHRP28 was detected by anti-BmPSI polyclonal antibody. Input represents proteins extracted from silkworm testis. Recombined BmPSI was obtained by prokaryotic over-expression.

\section{Construction of Y2H cDNA library of silkworm early embryo}

In B. mori, Bmdsx pre-mRNA undergoes default processing to generate the female Bmd$s x$ mRNA (Suzuki et al., 2001). The male-specific splicing of $B m d s x$ pre-mRNA may be regulated by BmPSI, Bmhrp28 and BmIMP (Wang et al., 2009; Suzuki et al., 2008, 2010). To explore whether there were other regulation factors to regulate Bmdsx in the BmHRP28 and BmPSI multiprotein complex, we constructed a Y2H cDNA library of early embryo. The white egg strain of the sex-limited silkworm (male) was used to extract mRNA for cDNA library construction. 
The cDNAs were connected to the pDONR222 vector to generate a Gateway entry library. The entry library was then transferred into pDEST 22 vector to obtain the two-hybrid cDNA library. The total number of clones in the library was $1.36 \times 10^{7}$. Randomly, we picked out 40 colonies and extracted plasmid DNA for PCR detection. The results showed that the average size of insert fragments was larger than $1.3 \mathrm{~kb}$ and that the recombination rate was greater than $95 \%$ (Figure 4). We diluted $1 \mu \mathrm{L}$ cDNA library $10^{6}$ times and spread it onto plates. After culture, 77 colonies grew. We calculated that the library titer was $7.7 \times 10^{10} \mathrm{CFU} / \mathrm{mL}$. These results showed that the library could be used for the library screening.

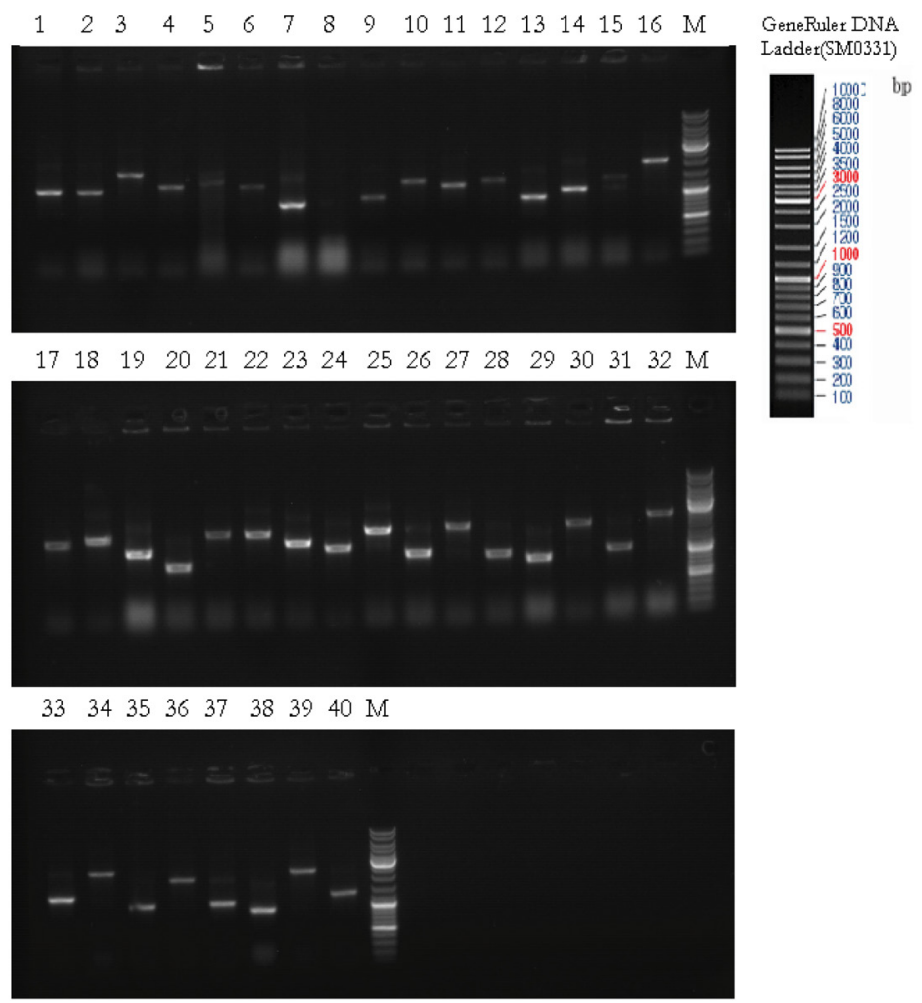

Figure 4. Quality of yeast two-hybrid cDNA library of silkworm early embryo. The average insert size and percentage of recombination of primary library. We randomly picked out 40 transformants from the plate, and their plasmids were isolated and retransformed into Escherichia coli. Subsequently, the clones containing "prey" plasmids were obtained and their sizes were identified with PCR. Lanes 1-40= inserting segment of primary library; lane $M$ $=$ GeneRuler DNA ladder.

\section{Bmspx was identified to interact with BmPSI by $\mathrm{Y} 2 \mathrm{H}$}

To further identify other possible regulation factors in the regulatory complex, we performed a $\mathrm{Y} 2 \mathrm{H}$ assay. We constructed a hybrid plasmid pDBLeu-BmPSI, which later served as a bait plasmid for screening the Y2H cDNA library on the basis of a GAL4 Y2H. After colonies grew on SC-Trp-Leu-His plates with $30 \mathrm{mM} 3$-AT added for seven days, there were 22 transformants of yeast colonies on the plates, and the 15 th colony was particularly 
strong (Figure S3). We crossed on the SC-Trp-Leu plates to save the strain and picked out a small colony to detect expression of the reporter gene LacZ. LacZ-positive clones were detected by the SC-Trp-Leu-Ura plates. Prey plasmid was extracted from $L a c Z$ and $U R A 3$ yeast co-transformed with the bait plasmid for validation. Finally, only one positive clone (c15) was confirmed for interaction (Figure 5).

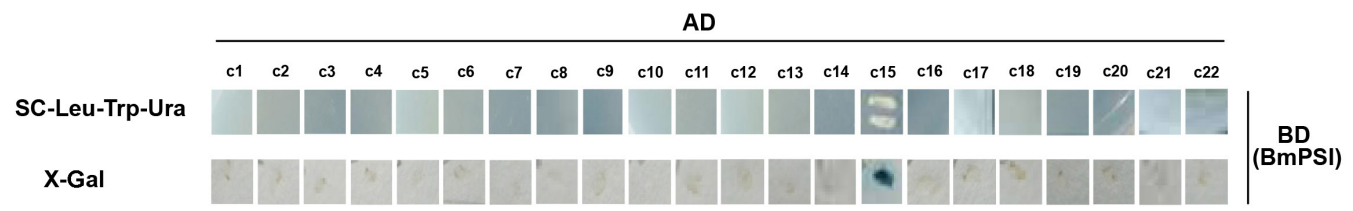

Figure 5. Identification of other regulation factors in the BmHRP28 and BmPSI regulator complexes by yeast twohybrid screen. The vector $\mathrm{pDBLeu}$-BmPSI was used as BD vector to test interaction with 22 positive clones from screen. $\mathrm{c} 1-\mathrm{c} 22=$ the number of positive clones. Results show that the clone No. 15 (c15) interacts with BmPSI.

The cloning and sequencing of this positive clone showed that this gene was homologous to a spliceosomal protein on the $\mathrm{X}$ chromosome of Drosophila (spx), and we named it B. mori spx (Bmspx). Domain prediction showed that this gene, which has two identical RRM domains like Bmhrp28, also encodes a RNA-binding protein. Compared with SPX protein sequence of Drosophila and SF3B of Apis mellifera, Mus musculus, Homo sapiens, and Danio rerio, BmSPX shows very high identity in the RRM domains (Figure 6). Shown by genomewide microarray expression data of tissues, Bmspx expresses in every tissue in fifth-instar day 3 but has the highest expression level in testis (Figure S4). Microarray expression data indicated that Bmspx may play a role in silkworm sperm development. However, the function of this gene in the sex determination of silkworm still needs to be studied.

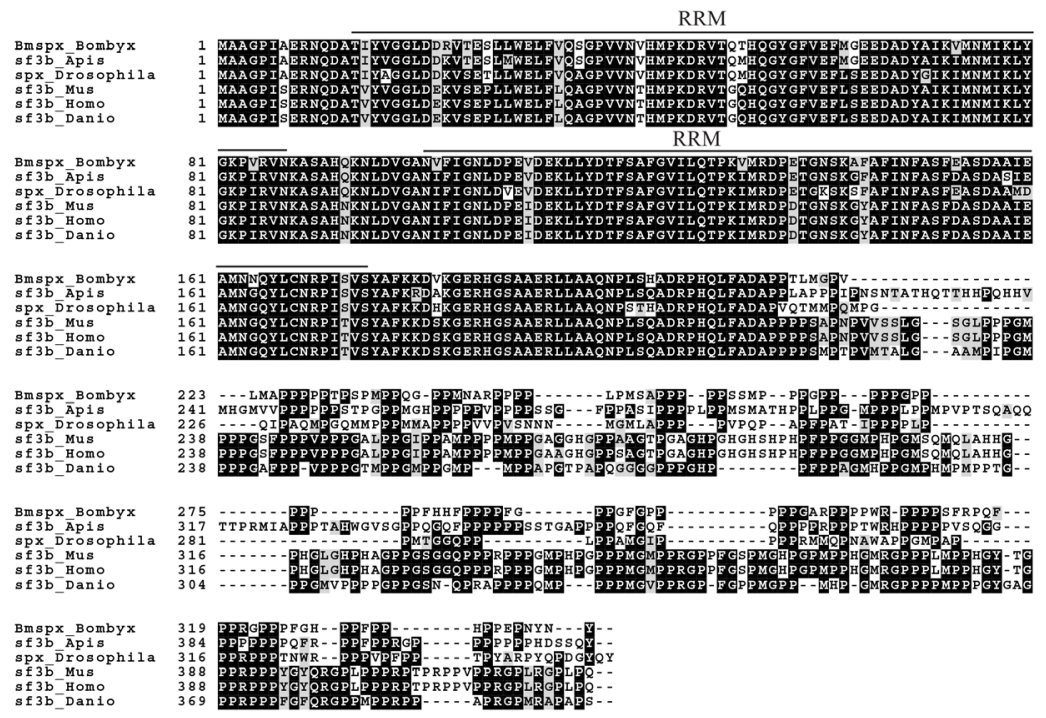

Figure 6. BmSPX is a conserved protein from insects to human. Multiple sequence alignment was performed with CLUSTALX and amino-acid shading with BOXSHADE 3.21 (http://www.ch.embnet.org/software/BOX_form.html). Black and grey indicate identical and similar amino acids, respectively. Beeline show two RRM domains. The species are Bombyx mori, Apis mellifera, Drosophila melanogaster, Mus musculus, Homo sapiens, and Danio rerio. 


\section{DISCUSSION}

To better understand the relationship between BmPSI and Bmhrp28, we utilized Y2H and Co-IP to assess the interaction of BmPSI with Bmhrp28. We found that after bait protein BmPSI and prey protein BmHRP28 were co-transfected into yeast, the expressions of the three reporter genes HIS3, URA3, LacZ were not detected. This result demonstrated that there was little or no direct binding between BmPSI and Bmhrp 28 (Figure 2). Co-IP protein interaction assays have shown that BmPSI and BmHRP28 coexist in the testis extract, which precipitated with anti-BmHRP28 antibody (Figure 3). In Drosophila, the tissue-specificity of P-element transposition is regulated at the level of pre-mRNA splicing (Laski et al., 1986). In the soma, a multiprotein complex binds to a 5 ' exon inhibitory element and appears to repress splicing of the Drosophila P-element third intron (IVS3). The two components of the multiprotein complex have been identified, PSI and hrp 48 (Siebel et al., 1994). PSI and hrp 48 are soma-specific alternative splicing factors, which are necessary for inhibition of IVS3 splicing (Siebel et al., 1994; Hammond et al., 1997). In this process, PSI cooperates with hrp48 to inhibit the splicing of Drosophila P element IVS3 in somatic cells (Siebel et al., 1994; Adams et al., 1997). In Bombyx mori, previous research showed that the Bombyx homologue of Drosophila PSI, BmPSI may work with the Bmhrp 28 together to recruit the U1 snRNP to the $5^{\prime}$ splice-site-like sequence and block the splicing of the $3^{\prime}$ splice site at intron 3 of Bmdsx, leading to the exclusion of exon 4 in the male (Suzuki et al., 2008; Wang et al., 2009). Along with the findings of our study, it is reasonable to speculate that the BmPSI may cooperate with Bmhrp 28 together as a multiprotein complex, but not with direct binding.

Are there any other regulation factors in the multiprotein complex that includes BmPSI and Bmhrp28? The evidence suggested that the alternative splicing system of pre-mRNAs contain several regulatory factors (Lopez, 1998). Recently, another male-specific RNAbinding protein, BmIMP, was identified, which interacts with BmPSI and increases BmPSICE1 RNA-binding activity (Suzuki et al., 2010). In this study, we constructed a Y2H cDNA library (Figure 4) from male early embryo of silkworm, and screened it with BmPSI to look for other factors in the complex. We found a protein from the cDNA library that could directly bind to the bait protein BmPSI (Figure 5). This protein was characterized and named BmSPX.

Sequence analysis showed that Bmspx, which has two identical RRM domains like Bmhrp28, is also an RNA-binding protein. Sequence alignment revealed that BmSPX is conserved with SPX protein of Drosophila and SF3B of A. mellifera, M. musculus, H. sapiens, D. rerio, etc. (Figure 6). The splicing factor SF3B is a U2 snRNP-associated protein complex essential for spliceosome assembly. The evidence showed that SF3B includes the spliceosomal proteins SAPs 49, 130, 145, and 155 (Das et al., 1999). These spliceosomal proteins are components of $17 \mathrm{~S}$ U2 snRNP. In mammals, functional 17S U2 snRNP can be assembled from 12S U2 snRNP and two essential splicing factors, SF3A and SF3B (Brosi et al., 1993a,b). Spliceosome assembly is initiated by the interaction of the U1 snRNP with the 5' splice site, forming the E complex. The latter also contains the 17S U2 snRNP, which at this stage associates via a non-base pairing interaction (Das et al., 2000). Therefore, our findings together with previous reports (Wang et al., 2009; Suzuki et al., 2008, 2010) suggest that BmSPX as a member of spliceosomal proteins works with BmPSI and BmHRP28 to build a spliceosome complex and thereby regulates $B m d s x$ in sex determination in the silkworm (Figure 7). Indeed, further research is needed to address whether BmSPX binds to BmHRP28 in the protein complex and whether there are other factors involved in their interaction. 


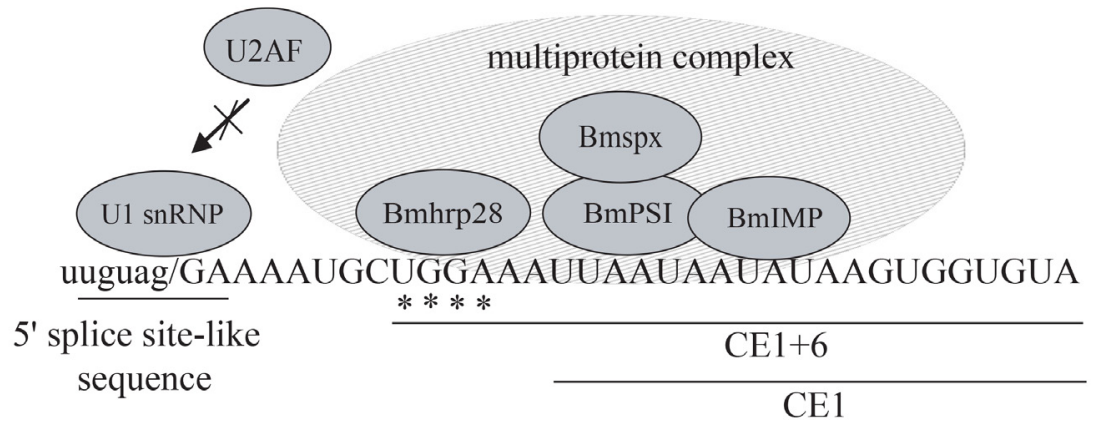

Figure 7. A possible model of $B m d s x$ spliceosome complex. The thick lines represent the sequences of CE1 and CE1+6 of Bmdsx. The slash represents 3' splice site. The asterisks indicate the sequence resembling the core sequence UAG (G/A) of hnRNP A1 binding sites. Rencently, BmIMP was indentified to interact with BmPSI (Suzuki et al., 2010). BmPSI and BmHRP28 coexist in a spliceosome complex on the Bmdsx exon 4, but not directly bind. Newly identified protein BmSPX interacts with BmPSI. They act together to recruit the U1 snRNP bind to a $5^{\prime}$ splice site-like sequence lied within the $3^{\prime}$ splice site, which blocks the binding of general splicing factors, such as U2AF, to the $3^{\prime}$ splice site, then preventing the splicing on this site.

\section{ACKNOWLEDGMENTS}

Research supported by the National Basic Research Program of China (\#2012CB114600), the National Hi-Tech Research and Development Program of China (\#2011AA00306), and the National Natural Science Foundation of China (\#31272502).

\section{Supplementary material}

\section{REFERENCES}

Adams MD, Tarng RS and Rio DC (1997). The alternative splicing factor PSI regulates P-element third intron splicing in vivo. Genes Dev. 11: 129-138.

Baker BS (1989). Sex in flies: the splice of life. Nature 340: 521-524.

Baker BS and Wolfner MF (1988). A molecular analysis of doublesex, a bifunctional gene that controls both male and female sexual differentiation in Drosophila melanogaster. Genes Dev. 2: 477-489.

Baker BS, Burtis K, Goralski T, Mattox W, et al. (1989). Molecular genetic aspects of sex determination in Drosophila melanogaster. Genome 31: 638-645.

Brosi R, Hauri HP and Kramer A (1993a). Separation of splicing factor SF3 into two components and purification of SF3a activity. J. Biol. Chem. 268: 17640-17646.

Brosi R, Groning K, Behrens SE, Luhrmann R, et al. (1993b). Interaction of mammalian splicing factor SF3a with U2 snRNP and relation of its 60-kD subunit to yeast PRP9. Science 262: 102-105.

Burtis KC and Baker BS (1989). Drosophila doublesex gene controls somatic sexual differentiation by producing alternatively spliced mRNAs encoding related sex-specific polypeptides. Cell 56: 997-1010.

Cline TW (1989). The affairs of daughterless and the promiscuity of developmental regulators. Cell 59: 231-234.

Cline TW (1993). The Drosophila sex determination signal: how do flies count to two? Trends Genet. 9: 385-390.

Cline TW and Meyer BJ (1996). Vive la difference: males vs females in flies vs worms. Annu. Rev. Genet. 30: 637-702.

Das BK, Xia L, Palandjian L, Gozani O, et al. (1999). Characterization of a protein complex containing spliceosomal proteins SAPs 49, 130, 145, and 155. Mol. Cell Biol. 19: 6796-6802.

Das R, Zhou Z and Reed R (2000). Functional association of U2 snRNP with the ATP-independent spliceosomal complex E. Mol. Cell 5: 779-787. 
Hammond LE, Rudner DZ, Kanaar R and Rio DC (1997). Mutations in the hrp48 gene, which encodes a Drosophila heterogeneous nuclear ribonucleoprotein particle protein, cause lethality and developmental defects and affect P-element third-intron splicing in vivo. Mol. Cell Biol. 17: 7260-7267.

Hashimoto H (1933). The role of the W chromosome in the sex determination of Bombyx mori. Jpn. J. Genet. 8: 245-258.

Inoue K, Hoshijima K, Higuchi I, Sakamoto H, et al. (1992). Binding of the Drosophila transformer and transformer-2 proteins to the regulatory elements of doublesex primary transcript for sex-specific RNA processing. Proc. Natl. Acad. Sci. U. S. A. 89: 8092-8096.

Laski FA, Rio DC and Rubin GM (1986). Tissue specificity of Drosophila P element transposition is regulated at the level of mRNA splicing. Cell 44: 7-19.

Lopez AJ (1998). Alternative splicing of pre-mRNA: developmental consequences and mechanisms of regulation. Annu. Rev. Genet. 32: 279-305.

Ohbayashi F, Suzuki MG, Mita K, Okano K, et al. (2001). A homologue of the Drosophila doublesex gene is transcribed into sex-specific mRNA isoforms in the silkworm, Bombyx mori. Comp. Biochem. Physiol. B Biochem. Mol. Biol. 128: $145-158$.

Schutt C and Nothiger R (2000). Structure, function and evolution of sex-determining systems in Dipteran insects. Development 127: 667-677.

Siebel CW, Kanaar R and Rio DC (1994). Regulation of tissue-specific P-element pre-mRNA splicing requires the RNAbinding protein PSI. Genes Dev. 8: 1713-1725.

Slee R and Bownes M (1990). Sex determination in Drosophila melanogaster. Q Rev. Biol. 65: 175-204.

Steinmann-Zwicky M, Amrein H and Nothiger R (1990). Genetic control of sex determination in Drosophila. Adv. Genet. 27: 189-237.

Suzuki MG, Ohbayashi F, Mita K and Shimada T (2001). The mechanism of sex-specific splicing at the doublesex gene is different between Drosophila melanogaster and Bombyx mori. Insect Biochem. Mol. Biol. 31: 1201-1211.

Suzuki MG, Funaguma S, Kanda T, Tamura T, et al. (2003). Analysis of the biological functions of a doublesex homologue in Bombyx mori. Dev. Genes Evol. 213: 345-354.

Suzuki MG, Funaguma S, Kanda T, Tamura T, et al. (2005). Role of the male BmDSX protein in the sexual differentiation of Bombyx mori. Evol. Dev. 7: 58-68.

Suzuki MG, Imanishi S, Dohmae N, Nishimura T, et al. (2008). Establishment of a novel in vivo sex-specific splicing assay system to identify a trans-acting factor that negatively regulates splicing of Bombyx mori dsx female exons. Mol. Cell Biol. 28: 333-343.

Suzuki MG, Imanishi S, Dohmae N, Asanuma M, et al. (2010). Identification of a male-specific RNA binding protein that regulates sex-specific splicing of Bmdsx by increasing RNA binding activity of BmPSI. Mol. Cell Biol. 30: 5776-5786.

Tian M and Maniatis T (1993). A splicing enhancer complex controls alternative splicing of doublesex pre-mRNA. Cell 74: 105-114.

Wang Z, Zhao M, Li D, Zha X, et al. (2009). BmHrp28 is a RNA-binding protein that binds to the female-specific exon 4 of Bombyx mori dsx pre-mRNA. Insect Mol. Biol. 18: 795-803. 